\title{
Evolution of spin excitations into the superconducting state in $\mathrm{FeTe}_{1-x} \mathrm{Se}_{x}$
}

\author{
M. D. Lumsden ${ }^{1 \star}$, A. D. Christianson ${ }^{1 \star}$, E. A. Goremychkin ${ }^{2,3}$, S. E. Nagler ${ }^{1}$, H. A. Mook ${ }^{1}$, M. B. Stone ${ }^{1}$, \\ D. L. Abernathy ${ }^{1}$, T. Guidi ${ }^{3}$, G. J. MacDougall' ${ }^{1}$ C. de la Cruz ${ }^{4}$, A. S. Sefat ${ }^{1}$, M. A. McGuire ${ }^{1}$, B. C. Sales ${ }^{1}$ \\ and D. Mandrus ${ }^{1}$
}

The origin of the superconducting state in the recently discovered Fe-based materials ${ }^{1-3}$ is the subject of intense scrutiny. Neutron scattering ${ }^{4-7}$ and NMR (ref. 8) measurements have already demonstrated a strong correlation between magnetism and superconductivity. A central unanswered question concerns the nature of the normal-state spin fluctuations that may be responsible for the pairing. Here we present inelastic neutron scattering measurements from large single crystals of superconducting and non-superconducting $\mathrm{Fe}_{1+y} \mathrm{Te}_{1-x} \mathrm{Se}_{x}$. These measurements indicate a spin fluctuation spectrum dominated by two-dimensional incommensurate excitations extending to energies greater than $250 \mathrm{meV}$. Most importantly, the spin excitations in $\mathrm{Fe}_{1+y} \mathrm{Te}_{1_{-}} \mathrm{Se}_{x}$ have four-fold symmetry about the $(1,0)$ wavevector (square-lattice $(\pi, \pi)$ point). Moreover, the excitations are described by the identical wavevector and can be characterized by the same model as the normal-state spin excitations in the high- $T_{C}$ cuprates $^{9-11}$. These results demonstrate commonality between the magnetism in these classes of materials, which perhaps extends to a common origin for superconductivity.

The discovery of superconductivity in $\mathrm{LaFeAsO}_{1-x} \mathrm{~F}_{x}$ with $T_{\mathrm{C}}=28 \mathrm{~K}$ (ref. 1 ) sparked a flurry of scientific activity and $T_{\mathrm{C}}$ rapidly increased to $\sim 55 \mathrm{~K}$ on replacing $\mathrm{La}$ with other rare-earth elements ${ }^{2,12,13}$. In addition to the $\mathrm{RFeAsO}$ family of compounds, superconductivity was also discovered in $\mathrm{AFe}_{2} \mathrm{As}_{2}$ (ref. 3), LiFeAs (ref. 14), and in the alpha phase of $\mathrm{Fe}_{1+y} \mathrm{Te}_{1-x} \mathrm{Se}_{x}$ (refs 15, 16). These materials share common structural square layers with $\mathrm{Fe}$ coordinated with either a pnictogen or a chalcogen. The unit cell contains two $\mathrm{Fe}$ atoms generating a reciprocal space rotated by $45^{\circ}$ from the conventional square lattice (see Fig. 1a). In $\mathrm{RFeAsO}$ and $\mathrm{AFe}_{2} \mathrm{As}_{2}$, the parent compounds show long-range spin-density-wave order characterized by the wavevector $\mathbf{Q}=(1 / 2,1 / 2, L)$ (refs 17-19). Doping suppresses magnetic order, allowing superconductivity to emerge with the concomitant appearance of a resonance in the spin fluctuation spectrum $^{4-7}$. However, the resonance probably contains only a small fraction of the total magnetic spectral weight and, consequently, understanding the role magnetism has in the superconductivity requires a detailed understanding of the higher energy spectrum of magnetic excitations.

The $\mathrm{Fe}_{1+y} \mathrm{Te}_{1-x} \mathrm{Se}_{x}$ materials are ideal candidates for a study of the magnetic excitations as large single crystals, necessary for detailed inelastic neutron scattering studies, may be grown. However, these materials differ from other Fe-based superconductors in that the $\mathrm{Fe}_{1+y}$ Te endpoint member (for small $y$ ) orders magnetically with a structure described by the wavevector $(1 / 2,0,1 / 2)$ (ref. 20) as opposed to $(1 / 2,1 / 2, L)$. Despite this difference, superconducting samples of $\mathrm{Fe}_{1+y} \mathrm{Te}_{1-x} \mathrm{Se}_{x}$ with higher Se content show a magnetic resonance at the same $(1 / 2,1 / 2)$ wavevector as other Fe-based superconductors $^{7,21}$ suggesting commonality in the magnetic response. To explore the magnetic excitations, inelastic neutron scattering measurements were carried out using the MERLIN spectrometer at the ISIS neutron scattering facility and the ARCS spectrometer at the Spallation Neutron Source. Measurements of lower energy excitations were carried out using the HB1 and HB3 triple-axis spectrometers at the High Flux Isotope Reactor. The single-crystal samples of $\mathrm{Fe}_{1.04} \mathrm{Te}_{0.73} \mathrm{Se}_{0.27}$ and $\mathrm{FeTe}_{0.51} \mathrm{Se}_{0.49}$ studied here were prepared as in ref. 22. Bulk measurements indicate weak, probably filamentary, superconductivity in $\mathrm{Fe}_{1.04} \mathrm{Te}_{0.73} \mathrm{Se}_{0.27}$ and bulk superconductivity in $\mathrm{FeTe}_{0.51} \mathrm{Se}_{0.49}$. Neither sample shows long-range magnetic order; however, for $x=0.27$, short-range magnetic order is observed ${ }^{20}$.

Figure $2 \mathrm{a}-\mathrm{h}$ summarizes the measured magnetic excitations at several energy transfers for both the $x=0.27$ and $x=0.49$ samples. We first note that the observed spectrum of magnetic excitations is two-dimensional (2D) in nature. Measurements at multiple sample-rotation angles were combined to allow for examination of the $L$ dependence at fixed $(H, K)$ coordinates. Such measurements indicate intensity that varies only weakly with $L$ (see Supplementary Information), as expected for 2D excitations. This is consistent with recent measurements of a $2 \mathrm{D}$ magnetic resonance in $\mathrm{FeSe}_{0.4} \mathrm{Te}_{0.6}$ (ref. 21).

The low-energy magnetic response (Fig. 2a,b) for the $x=0.27$ sample is characterized by two peaks at wavevectors near $(1 / 2,1 / 2)$. Interestingly, the data do not show four-fold symmetry around this wavevector as expected for the nuclear cell of the $P 4 / \mathrm{nmm}$ space group, but rather form a quartet around the $(1,0)$ wavevector. With increasing energy, the peaks disperse away from $(1 / 2,1 / 2)$ towards $(1,0)$ as shown schematically in Fig. $1 \mathrm{~b}$. At higher energies, the excitations continue to disperse towards $(1,0)$ but evolve from spots into rings (Fig. 2c) centred on this wavevector. Eventually, as shown for an energy transfer of $120 \mathrm{meV}$ in Fig. 2d, the excitations evolve into broad spots centred at $(1,0)$. For the superconducting $x=0.49$ sample, the low-energy spectrum appears as a series of asymmetric spots (Fig. 2e). However, data measured at a higher energy transfer of $22 \mathrm{meV}$ (Fig. 2f) show separated peaks similar to those in the $x=0.27$ sample. This can easily be understood by considering that the displacement of these peaks away from $(1,0)$ is larger in the

${ }^{1}$ Oak Ridge National Laboratory, Oak Ridge, Tennessee 37831, USA, ${ }^{2}$ Argonne National Laboratory, Argonne, Illinois 60439, USA, ${ }^{3}$ ISIS Facility, Rutherford Appleton Laboratory, Chilton, Didcot OX11 0QX, UK, ${ }^{4}$ The University of Tennessee, Knoxville, Tennessee 37996, USA. *e-mail: lumsdenmd@ornl.gov; postfab@googlemail.com. 


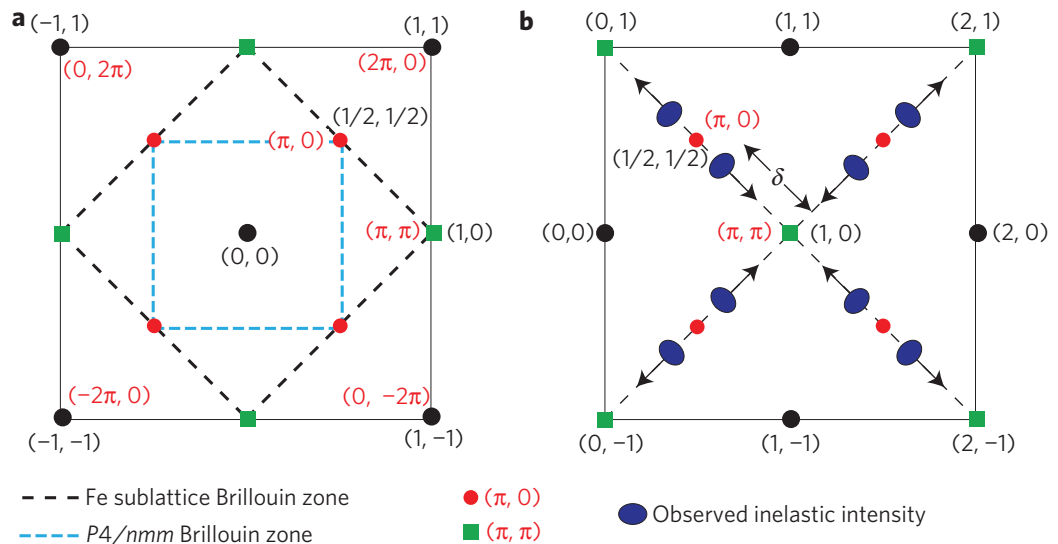

Figure 1 | Reciprocal space for $\mathbf{F e}_{1+\boldsymbol{y}} \mathbf{T e}_{\mathbf{1}} \mathbf{S} \mathbf{S e}_{\mathbf{x}}$ compounds. The black (red) labels represent wavevectors in the tetragonal (square) reciprocal lattice. The red circles indicate the square-lattice $(\pi, 0)$ points where the resonance is observed and the green squares indicate the square-lattice $(\pi, \pi)$ points. $\mathbf{a}$, The dashed blue line denotes the Brillouin zone of the $P 4 / n m m$ crystal structure. For the Fe sublattice, $(1,0)$ is no longer a zone centre and the Brillouin zone becomes larger, as represented by the black dashed line. $\mathbf{b}$, The same diagram as in $\mathbf{a}$, but centred at $(1,0)$. The blue ovals show the location of the observed excitations and the arrows represent the direction of dispersion with increasing energy transfer. The distance from $(1,0)$ is indicated by $\delta$.
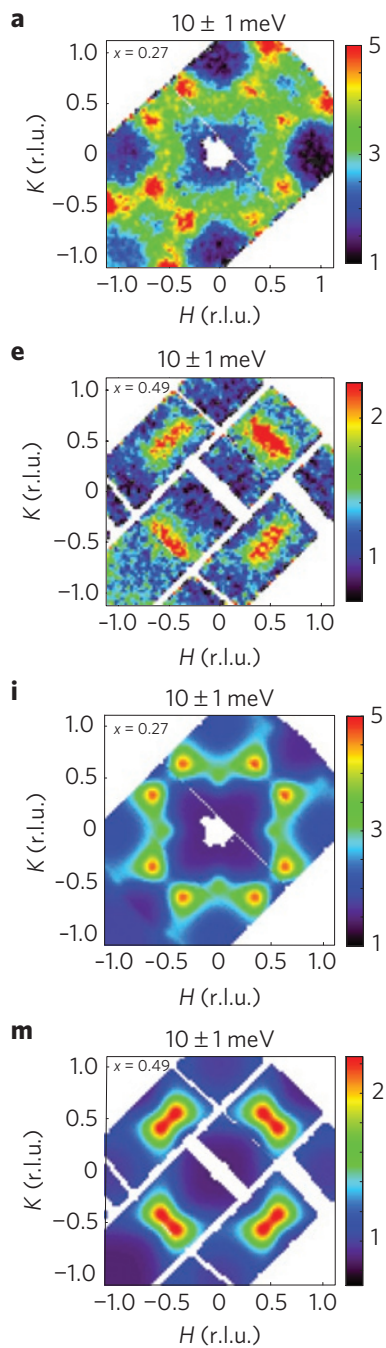

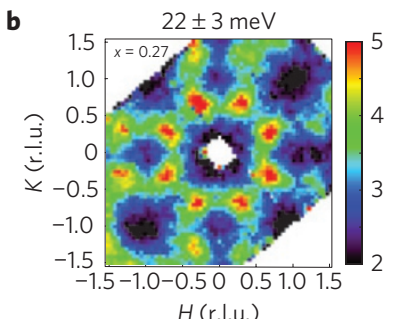

$\mathbf{f}$
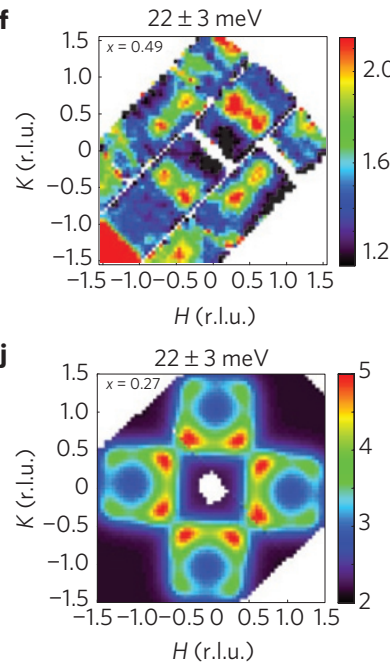

n

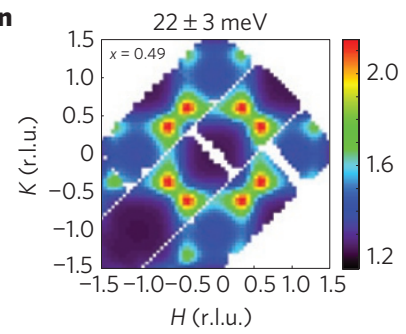

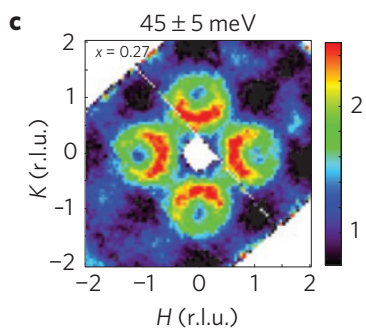
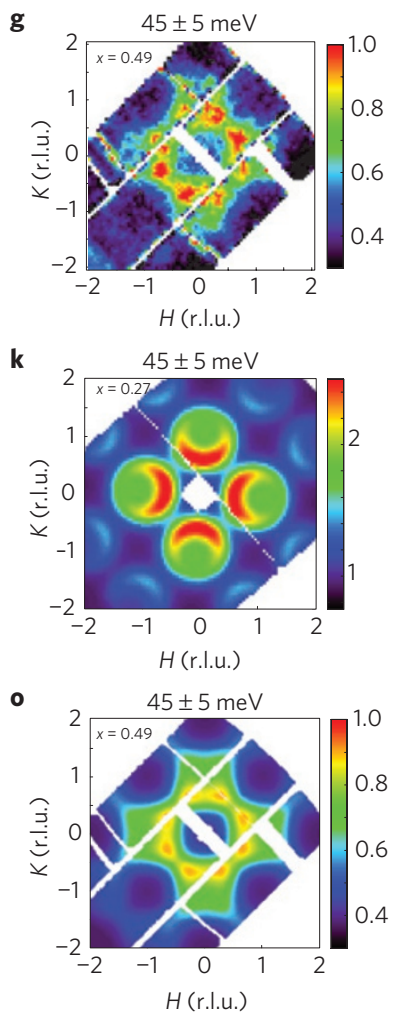

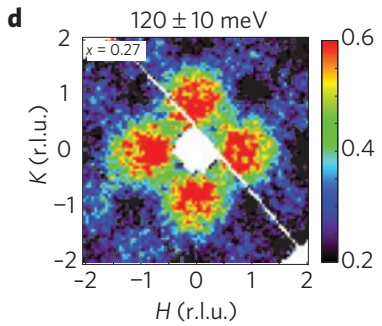

h
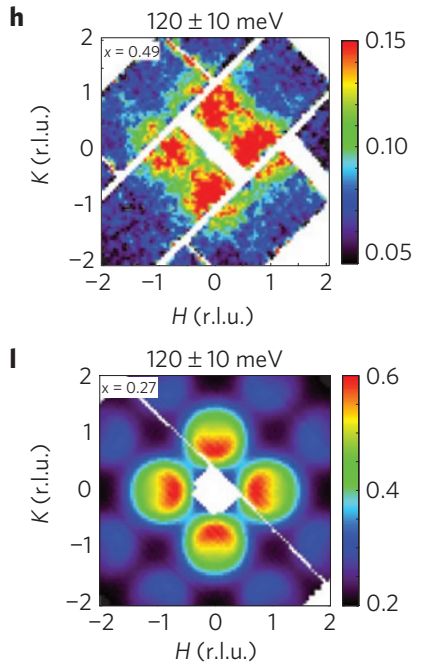

p

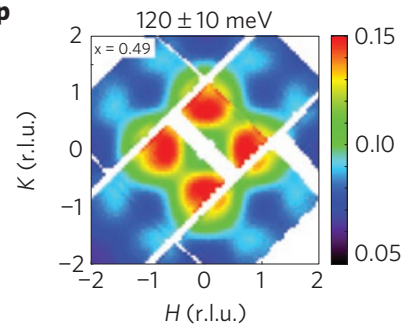

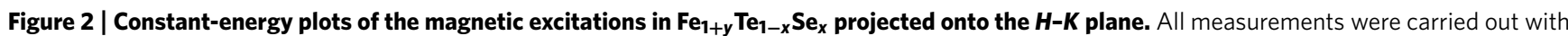
the $c$ axis parallel to the incident beam with a sample temperature of 5 (3.5) K for $x=0.27$ (0.49). a-d, Data from the $x=0.27$ sample measured using MERLIN with incident energies of 25 (a), 60 (b), 120 (c) and $250 \mathrm{meV}(\mathbf{d})$. e-h, Data from the $x=0.49$ sample measured using ARCS with incident energies of $40(\mathbf{e}), 60(\mathbf{f}), 120(\mathbf{g})$ and $250 \mathrm{meV}(\mathbf{h})$. The $x=0.27(0.49)$ samples were single crystals with a mass of 16.91 (15.45) g. i-p, Fits to equation (1) convolved with instrumental resolution for the $x=0.27$ (i-I) and $x=0.49$ sample (m-p). The fit (i) to the 10 meV data (a) for the $x=0.27$ sample includes two components rotated by $45^{\circ}$ in the $\mathrm{H}-\mathrm{K}$ plane. All other fits include only a single component. 


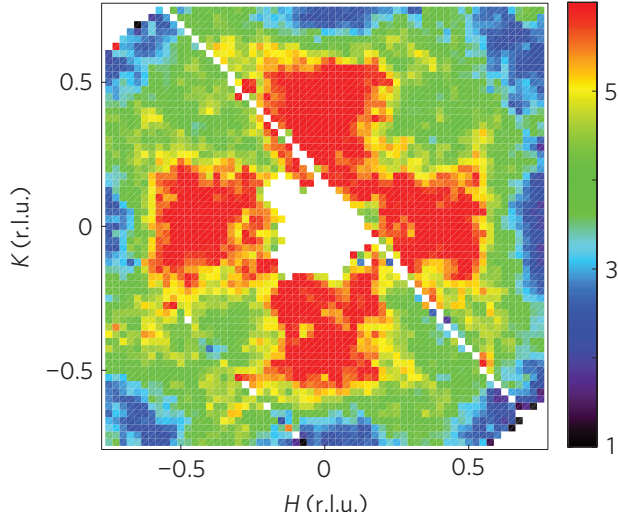

c

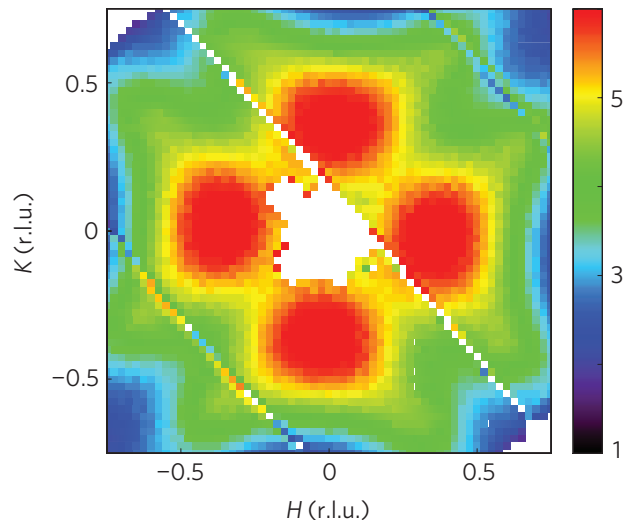

b

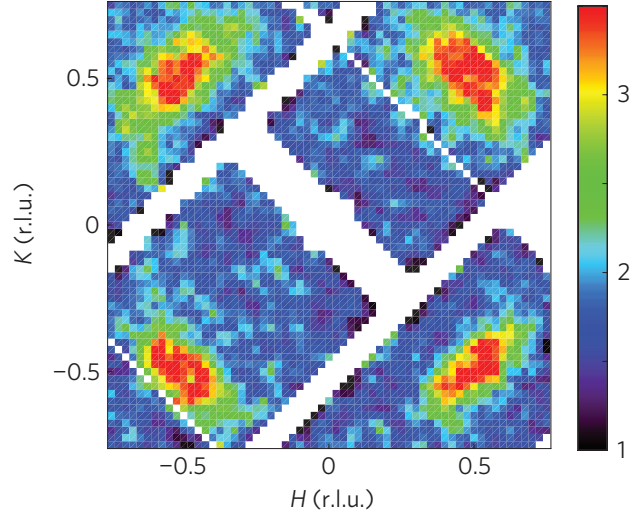

d

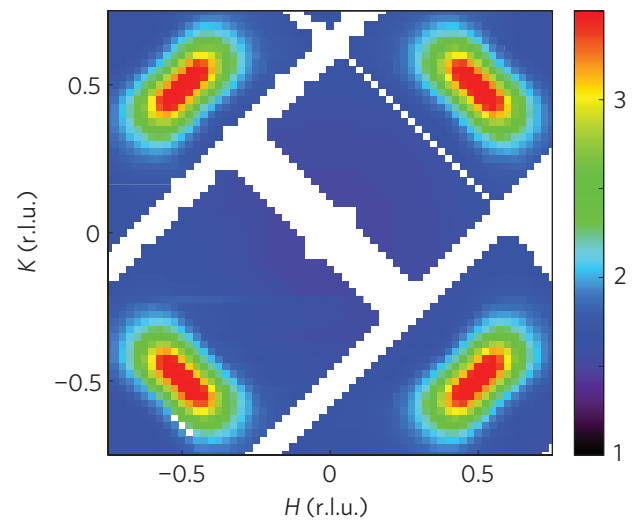

Figure $\mathbf{3}$ | Constant-energy plots of the magnetic excitation spectrum at an energy transfer of $\mathbf{6} \pm \mathbf{1} \mathbf{m e V}$. $\mathbf{a}, \mathbf{b}$, Measurements of the $x=0.27$ (a) and $x=0.49$ sample (b) were carried out with an incident energy of 25 and $40 \mathrm{meV}$, respectively. The temperature was $5(3.5) \mathrm{K}$ for the $x=0.27(0.49)$ measurements. These data show an extra component in the $x=0.27$ data centred near $(1 / 2,0)$, which is absent in the $x=0.49$ data. $\mathbf{c}, \mathbf{d}$, Fits to equation (1) convolved with instrumental resolution. Fits to the $x=0.27$ data (c) include two components rotated by $45^{\circ}$ with respect to one another in the $H-K$ plane, whereas fits to the $x=0.49$ data (d) include only a single, incommensurate component.

$x=0.49$ sample such that the pair of peaks around $(1 / 2,1 / 2)$ have moved closer together and overlap significantly. At an energy of $45 \mathrm{meV}$ (Fig. $2 \mathrm{~g}$ ), the characteristic wavevector appears similar in the two samples but the $x=0.49$ scattering appears to have not fully evolved into the rings of scattering present in the $x=0.27$ sample (Fig. 2c). At high energies, the scattering is similar in the two samples, as can be seen by comparing Fig. $2 \mathrm{~d}$ and h. The excitations persist to energy transfers greater than $250 \mathrm{meV}$ (see Supplementary Information) with Q-dependence similar to that shown at $120 \mathrm{meV}$ for all higher energies.

Examination of the wavevector describing these excitations reveals similarities with the high- $T_{\mathrm{C}}$ cuprates. The quartet of peaks is characterized by wavevectors $(1 \pm \xi, \pm \xi)$ and $(1 \pm \xi, \mp \xi)$ that, in square-lattice notation, corresponds to $(\pi \pm \xi, \pi)$ and $(\pi, \pi \pm \xi)$ as shown in Fig. 1b. This is precisely the same wavevector as the low-energy excitations observed in $\mathrm{La}_{2-x} \mathrm{Sr}_{x} \mathrm{CuO}_{4}$ (ref. 9) and $\mathrm{YBa}_{2} \mathrm{Cu}_{3} \mathrm{O}_{6+\mathrm{x}}$ (refs 10,11 ), indicating remarkable commonality in the excitation spectrum of these two classes of high- $T_{\mathrm{C}}$ superconductors. Furthermore, the evolution of the scattering from well-defined peaks at low energies to broadened rings at higher energies is a characteristic property of magnetic excitations in the cuprates $^{23,24}$. The magnitude of $\xi$, however, is much larger in $\mathrm{Fe}_{1+y} \mathrm{Te}_{1-x} \mathrm{Se}_{x}$, resulting in low-energy excitations displaced away from $(1,0)$ and much closer to $(1 / 2,1 / 2)$.

At low energies, the largest difference between the two concentrations becomes evident as shown in Fig. 3a,b. In addition to the excitations near $(1 / 2,1 / 2)$, an extra component centred near $(1 / 2,0)$ is present in the $x=0.27$ sample (also visible in Fig. 2a). With decreasing energy, the intensity of this component increases, eventually forming the short-range order observed previously for samples with a similar concentration ${ }^{20,25}$. It has been suggested ${ }^{26}$ that excess Fe in Te-rich samples results in local moments that may provide a pair-breaking mechanism destroying superconductivity. The component of scattering observed near $(1 / 2,0)$ is absent in the $x=0.49$ sample with no excess Fe (Fig. 3b). Furthermore, the scattering near $(1 / 2,0)$ exists inelastically for all energies below $\sim 10 \mathrm{meV}$ and, as such, exists well below the superconducting gap, potentially providing a pair-breaking mechanism. These observations are consistent with the extra component near $(1 / 2,0)$ existing as a result of the influence of extra Fe in the $x=0.27$ sample.

To quantify the dispersion of the spin excitations, the data were fitted using the phenomenological Sato-Maki function ${ }^{27}$ previously used for the cuprates ${ }^{24}$

$$
\chi^{\prime \prime}(\mathbf{Q}, \omega)=\chi_{0}(\omega) \frac{\kappa^{4}(\omega)}{\left[\kappa(\omega)^{2}+R(\mathbf{Q})\right]^{2}}
$$

where

$$
R(\mathbf{Q})=\frac{\left[\left(H-H_{\mathrm{C}}\right)^{2}+\left(K-K_{\mathrm{C}}\right)^{2}-\delta^{2}\right]^{2}+\frac{\lambda}{4}\left[\left(H-H_{\mathrm{C}}\right)^{2}-\left(K-K_{\mathrm{C}}\right)^{2}\right]^{2}}{4 \delta^{2}}
$$

The form for $R(\mathbf{Q})$ in equation (2) describes excitations fourfold symmetric about $\left(H_{\mathrm{C}}, K_{\mathrm{C}}\right)=(1,0)$. As discussed below, $\delta$ parameterizes the dispersion, $\lambda$ defines the evolution of the spectrum from peaks (large $\lambda$ ) to rings (small $\lambda$ ) and $\kappa$ is a broadening parameter.

The results of fits to the data presented in Fig. $2 \mathrm{a}-\mathrm{h}$ using the Sato-Maki function convolved with instrumental resolution are 
a

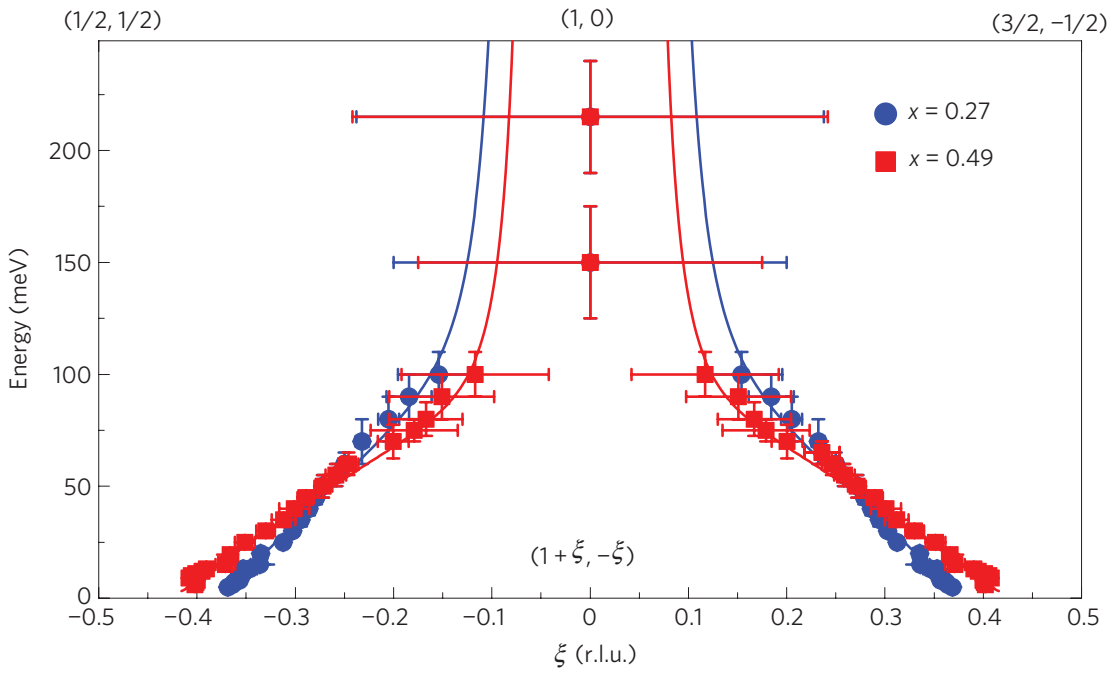

b
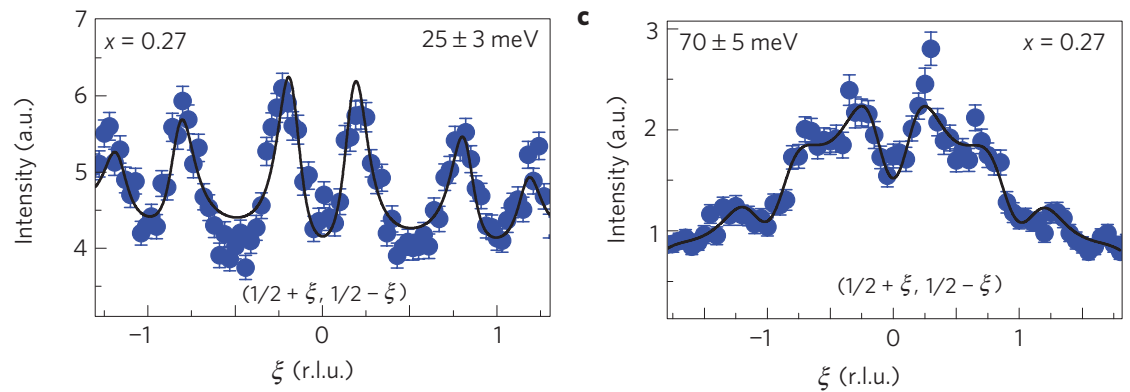

d
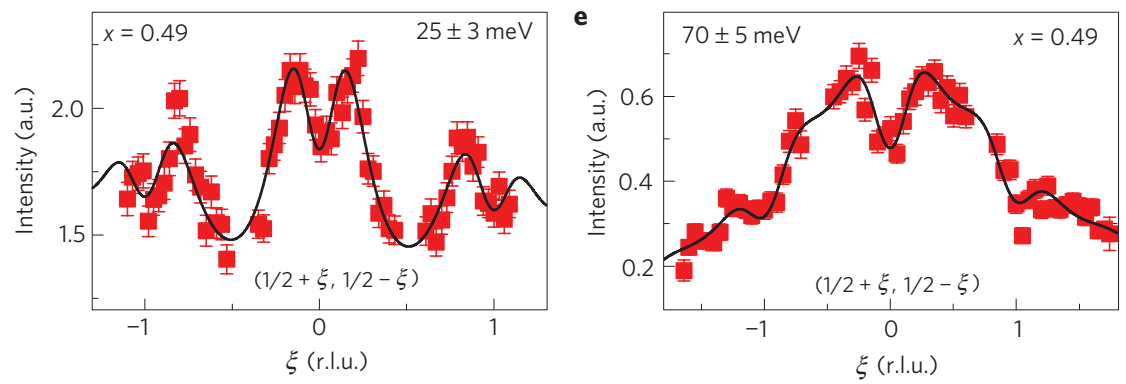

Figure 4 | Dispersion of the magnetic excitations in $\mathbf{F e}_{\mathbf{1 + y}} \mathbf{T} \mathbf{e}_{\mathbf{1}-\mathbf{x}} \mathbf{S} \mathbf{e}_{\mathbf{x}}$. Data at fixed energies are fitted using equation (1). $\mathbf{a}$, The dispersion along (1+ $\left.\xi,-\xi\right)$ is extracted from the best fit value of $\delta$ by noting that $\xi=\delta / \sqrt{ } 2$. Below $15 \mathrm{meV}$, the dispersion was extracted from triple-axis measurements in the (HKO) scattering plane through convolution of equation (1) with instrumental resolution. The dispersion above 15 meV was extracted from time-of-flight data. As instrumental resolution was found to have little effect on the fits to the time-of-flight data, the dispersion was generated without including resolution. For $x=0.27$, all data analysed were measured at $3.5 \mathrm{~K}$ (triple-axis) or $5 \mathrm{~K}$ (time-of-flight). For $x=0.49$, time-of-flight data analysed were measured at $3.5 \mathrm{~K}$, whereas triple-axis data were measured at $20 \mathrm{~K}$ to avoid scattering from the magnetic resonance. The horizontal error bars represent the statistical uncertainty in the fit to equation (1), whereas the vertical error bars represent the range of energies included. The solid lines in a are guides to the eye. For clarity, the dispersion is plotted for both positive and negative $\xi$, noting that equation (1) is an even function of $\delta$. Above 100 meV, the peak position cannot be distinguished from $\xi=0 . \mathbf{b}-\mathbf{e}$, Cuts at fixed energies obtained by integrating data on either side of the line $K=-H$ over $H= \pm 0.1$ in the perpendicular $(H, H)$ direction. The data were measured with incident energies of $120 \mathrm{meV}(\mathbf{b}, \mathbf{d})$ and $250 \mathrm{meV}(\mathbf{c}, \mathbf{e})$. The error bars in $\mathbf{b}$-e represent one sigma and solid lines are fits to equation (1).

shown in Fig. $2 \mathrm{i}-\mathrm{l}(\mathrm{m}-\mathrm{p})$ for $x=0.27(0.49)$. The fits agree well with the measurements over the full range of measured energies. The fit quality is also demonstrated in Fig. $4 \mathrm{~d}-\mathrm{g}$ for cuts along $(1 / 2 \pm \xi, 1 / 2 \mp \xi)$, indicating excellent agreement over a wide range of wavevector and energy transfer. A spin excitation spectrum accurately described by the identical model in both $\mathrm{Fe}_{1+y} \mathrm{Te}_{1-x} \mathrm{Se}_{x}$ and the cuprates is further evidence of commonality in these two classes of superconductor. The same Sato-Maki function can be used to describe the extra component in the $x=0.27$ sample near $(1 / 2,0)$ with a rotated $R(\mathbf{Q})$ factor (see Supplementary Information) and the fits shown in Figs $3 \mathrm{c}$ and $2 \mathrm{i}$ contain both components, again yielding excellent agreement with the data.
The best fit value of $\delta$ parameterizes the dispersion. If we define the characteristic wavevector as $\mathbf{q}_{m}=(1 \pm \xi, \mp \xi)$, then $\xi=\delta / \sqrt{ } 2$. The resulting dispersion (Fig. 4a) demonstrates excitations dispersing from a wavevector near $(1 / 2,1 / 2)(\xi=-0.5)$ towards $(1,0)(\xi=0)$. The shape of the dispersion is reminiscent of the 'hour glass' dispersion observed in the cuprates ${ }^{24,28}$. However, unlike the cuprates, the high-energy excitations of $\mathrm{Fe}_{1+y} \mathrm{Te}_{1-x} \mathrm{Se}_{x}$ remain centred near $(1,0)$ with no evidence for dispersion away from this wavevector. It is important to note that, for both concentrations, the dispersion does not approach the commensurate $(1 / 2,1 / 2)$ wavevector on approaching the elastic condition and, therefore, both samples show an incommensurate 
excitation spectrum. For energies greater than $\sim 50 \mathrm{meV}$, the dispersions are consistent for the two concentrations. However, for lower energies, $|\xi|$ is larger for $x=0.49$, indicating excitations displaced closer to the $(1 / 2,1 / 2)$ wavevector with larger Se content. This can also be clearly seen in comparing the cuts in Fig. $4 \mathrm{~b}$ with $4 \mathrm{~d}(25 \mathrm{meV})$ and $4 \mathrm{c}$ with $4 \mathrm{e}(70 \mathrm{meV})$. The peaks in the $25 \mathrm{meV}$ data are at clearly different wavevectors, but the distribution of scattering intensity is nearly identical at $70 \mathrm{meV}$ for the two concentrations. It is interesting to note that the sample $(x=0.49)$ with normal-state excitations closer to the resonance wavevector shows bulk superconductivity, whereas only weak superconductivity is observed in the sample with normal-state excitations displaced further from this wavevector.

Finally, we discuss the implications of the observed Q-dependence of the magnetic excitations. As a consequence of the coordination of the chalcogen atoms, the real-space $P 4 / \mathrm{nmm}$ unit cell contains two $\mathrm{Fe}$ atoms. On the other hand, as the system is not magnetically ordered, if one considers magnetism primarily associated with the $\mathrm{Fe}$ atoms, the effective magnetic unit cell is described by a $2 \mathrm{D}$ square lattice. The Brillouin zone of each of these lattices is illustrated in Fig. 1a. As seen in Fig. 2, the observed magnetic scattering shows periodicity compatible with the Fe sublattice Brillouin zone. This is consistent with the periodicity of spin fluctuations observed in the high- $T_{\mathrm{C}}$ cuprates, as expected because cuprate magnetism is dominated by $\mathrm{Cu}$ atoms that also form a square lattice. The essential magnetism in both $\mathrm{Fe}_{1+y} \mathrm{Te}_{1-x} \mathrm{Se}_{x}$ and the cuprates can be captured by the identical model even though the crystal structures themselves have different symmetries. We note that caution is necessary in comparing the Q-dependence of experimental data with theoretical calculations. Some published calculations have been presented in a 2D Brillouin zone based on the $P 4 / \mathrm{nmm}$ lattice. This Brillouin zone is smaller than the square-lattice Brillouin zone and, presented in this manner, calculations fold excitations in the zone centred at $(1$, $0)$ back to the zone centred at $(0,0)$ (ref. 29). This superposes the magnetic response in these two zones, making it difficult to extract the true $\mathbf{Q}$-dependence as measured experimentally. Calculations carried out in the square-lattice Brillouin zone should result in the symmetry of the measured excitation spectrum as confirmed by calculations of the $\mathbf{Q}$-dependent spin susceptibility and gap functions ${ }^{30}$.

\section{Received 24 July 2009; accepted 11 December 2009;} published online 17 January 2010

\section{References}

1. Kamihara, Y. et al. Iron-based layered superconductor $\mathrm{La}\left[\mathrm{O}_{1-x} \mathrm{~F}_{x}\right] \mathrm{FeAs}$ $(x=0.05-0.12)$ with $T_{\mathrm{C}}=26$ K. J. Am. Chem. Soc. 130, 3296-3297 (2008).

2. Chen, X. C. et al. Superconductivity at $43 \mathrm{~K}$ in $\mathrm{SmFeAsO}_{1_{-}} \mathrm{F}_{x}$. Nature 453, 761-762 (2008).

3. Rotter, M., Tegel, M. \& Johrendt, D. Superconductivity at $38 \mathrm{~K}$ in the iron arsenide $\left(\mathrm{Ba}_{1-x} \mathrm{~K}_{x}\right) \mathrm{Fe}_{2} \mathrm{As}_{2}$. Phys. Rev. Lett. 101, 107006 (2008).

4. Christianson, A. D. et al. Unconventional superconductivity in $\mathrm{Ba}_{0.6} \mathrm{~K}_{0.4} \mathrm{Fe}_{2} \mathrm{As}_{2}$ from inelastic neutron scattering. Nature 456, 930-932 (2008).

5. Lumsden, M. D. et al. Two-dimensional resonant magnetic excitation in $\mathrm{BaFe}_{1.84} \mathrm{Co}_{0.16} \mathrm{As}_{2}$. Phys. Rev. Lett. 102, 107005 (2009).

6. Chi, S. et al. Inelastic neutron-scattering measurements of a three-dimensional spin resonance in the FeAs-based $\mathrm{BaFe}_{1.9} \mathrm{Ni}_{0.1} \mathrm{As}_{2}$ superconductor. Phys. Rev. Lett. 102, 107006 (2009).

7. Mook, H. A. et al. Neutron scattering patterns show superconductivity in $\mathrm{FeTe}_{0.5} \mathrm{Se}_{0.5}$ likely results from itinerant electron fluctuations. Preprint at $<$ http://arxiv.org/abs/0904.2178vl> (2009).
8. Ning, F. et al. Spin susceptibility, phase diagram, and quantum criticality in the electron-doped high $\mathrm{T}_{\mathrm{C}}$ superconductor $\mathrm{Ba}\left(\mathrm{Fe}_{1-x} \mathrm{Co}_{x}\right) 2 \mathrm{As}_{2}$. J. Phys. Soc. Jpn 78, 013711 (2009).

9. Cheong, S.-W. et al. Incommensurate magnetic fluctuations in $\mathrm{La}_{2-x} \mathrm{Sr}_{x} \mathrm{CuO}_{4}$. Phys. Rev. Lett. 67, 1791-1794 (1991).

10. Dai, P., Mook, H. A. \& Dogan, F. Incommensurate magnetic fluctuations in $\mathrm{YBa}_{2} \mathrm{Cu}_{3} \mathrm{O}_{6.6}$. Phys. Rev. Lett. 80, 1738-1741 (1998).

11. Mook, H. A. et al. Spin fluctuations in $\mathrm{YBa}_{2} \mathrm{Cu}_{3} \mathrm{O}_{6.6}$. Nature 395, 580-582 (1998)

12. Chen, G. F. et al. Superconductivity at $41 \mathrm{~K}$ and its competition with spin-density-wave instability in layered $\mathrm{CeO}_{1-x} \mathrm{~F}_{x}$ FeAs. Phys. Rev. Lett. 100, 247002 (2008).

13. Ren, Z. et al. Superconductivity at $55 \mathrm{~K}$ in iron-based F-doped layered quaternary compound $\operatorname{Sm}\left[\mathrm{O}_{1-x} \mathrm{~F}_{x}\right]$ FeAs. Chin. Phys. Lett. 25, 2215-2216 (2008).

14. Wang, X. et al. The superconductivity at $18 \mathrm{~K}$ in LiFeAs system. Solid State Commun. 148, 538-540 (2008)

15. Hsu, F.-C. et al. Superconductivity in the PbO-type structure $\alpha$-FeSe. Proc. Natl Acad. Sci. USA 105, 14262-14264 (2008).

16. Yeh, K.-W. et al. Tellurium substitution effect on superconductivity of the $\alpha$-phase iron selenide. Europhys. Lett. 84, 37002 (2008).

17. de la Cruz, C. et al. Magnetic order close to superconductivity in the iron-based layered $\mathrm{LaO}_{1-x} \mathrm{~F}_{x} \mathrm{FeAs}$ systems. Nature 453, 899-902 (2008).

18. McGuire, M. A. et al. Phase transitions in LaFeAsO: Structural, magnetic, elastic, and transport properties, heat capacity and Mössbauer spectra. Phys. Rev. B 78, 094517 (2008).

19. Huang, Q. et al. Neutron-diffraction measurements of magnetic order and a structural transition in the parent $\mathrm{BaFe}_{2} \mathrm{As}_{2}$ compound of FeAs-based high-temperature superconductors. Phys. Rev. Lett. 101, 257003 (2008).

20. Bao, W. et al. Tunable $(\delta \pi, \delta \pi)$-type antiferromagnetic order in $\alpha$ - $\mathrm{Fe}(\mathrm{Te}, \mathrm{Se})$ superconductors. Phys. Rev. Lett. 102, 247001 (2009).

21. Qiu, Y. et al. Spin gap and resonance at the nesting wave vector in superconducting $\mathrm{FeSe}_{0.4} \mathrm{Te}_{0.6}$. Phys. Rev. Lett. 103, 067008 (2009).

22. Sales, B. C. et al. Bulk superconductivity at $14 \mathrm{~K}$ in single crystals of $\mathrm{Fe}_{1+y} \mathrm{Te}_{x} \mathrm{Se}_{1-x}$. Phys. Rev. B 79, 094521 (2009).

23. Stock, C. et al. From incommensurate to dispersive spin fluctuations: The high energy inelastic spectrum in superconducting $\mathrm{YBa}_{2} \mathrm{Cu}_{3} \mathrm{O}_{6.5}$. Phys. Rev. B 71, 024522 (2005).

24. Vignolle, B. et al. Two energy scales in the spin excitations of the high-temperature superconductor $\mathrm{La}_{2-x} \mathrm{Sr}_{x} \mathrm{CuO}_{4}$. Nature Phys. 3, 163-167 (2007).

25. Wen, J. et al. Coexistence and competition of short-range magnetic order and superconductivity in $\mathrm{Fe}_{1+\delta} \mathrm{Te}_{1-x} \mathrm{Se}_{x}$. Phys. Rev. B 80, 104506 (2009).

26. Zhang, L., Singh, D. J. \& Du, M. H. Density functional study of excess Fe in $\mathrm{Fe}_{1+x \mathrm{Te}}$ : Magnetism and doping. Phys. Rev. B 79, 012506 (2009).

27. Sato, H. \& Maki, K. Theory of inelastic neutron scattering from $\mathrm{Cr}$ and its alloys near the Néel temperature. Int. J. Magn. 6, 183-209 (1974).

28. Hayden, S. M. et al. The structure of the high-energy spin excitations in a high-transition-temperature superconductor. Nature 429, 531-534 (2004).

29. Mazin, I. I. et al. Unconventional superconductivity with a sign reversal in the order parameter of $\mathrm{LaFeAsO}_{1-x} \mathrm{~F}_{x}$. Phys. Rev. Lett. 101, 057003 (2008).

30. Kuroki, K. et al. Unconventional pairing originating from the disconnected fermi surfaces of superconducting $\mathrm{LaFeAsO}_{1-x} \mathrm{~F}_{x}$. Phys. Rev. Lett. 101, 087004 (2008).

\section{Acknowledgements}

We acknowledge discussions with D. Singh and T. Maier. This work was supported by the Scientific User Facilities Division and the Division of Materials Sciences and Engineering, Office of Basic Energy Sciences, US Department of Energy.

\section{Author contributions}

All authors made critical comments on the manuscript. M.D.L., A.D.C., E.A.G., S.E.N. M.B.S., D.L.A., T.G., G.J.M., C.C. and H.A.M. all contributed to data collection. A.S.S., M.A.M., B.C.S. and D.M. contributed to sample synthesis and characterization.

\section{Additional information}

The authors declare no competing financial interests. Supplementary information accompanies this paper on www.nature.com/naturephysics. Reprints and permissions information is available online at http://npg.nature.com/reprintsandpermissions. Correspondence and requests for materials should be addressed to M.D.L. or A.D.C. 